\title{
Predictive performance of transcranial Doppler for PDPH
}

\author{
Priyam Saikia ${ }^{1}$
}

Received: 25 June 2019 / Accepted: 24 August 2019 / Published online: 3 September 2019

(c) Japanese Society of Anesthesiologists 2019

Keywords Predictive performance $\cdot$ Pregnancy $\cdot$ Transcranial doppler

To the Editor:

I read with interest the article by Mowafy and Abd Ellatif and would like to bring a few points for due consideration [1].

1. Sample size should be based on the primary aim. Instead of using a predefined sensitivity/specificity or area under the curve (AUC) of receiver operating characteristic (ROC) curve, the authors chose to find an undisclosed difference of mean of cerebral artery velocity $[1,2]$. Moreover, the cited study does not report the values used by authors [1]. Thus, it is unclear whether their study was adequately powered to examine their primary aim.

2. The standard deviation (SD) of mean velocity and pulsatility index is very narrow compared to the difference between the means of the groups [1]. Previous study suggests some overlap of the dispersion of those two parameters between the groups $[1,3]$. This peculiar dispersion may have led to a 'significant' $p$ value, very narrow AUC of ROC and a near ideal sensitivity and specificity.

3. The reported $95 \%$ Confidence Interval (CI) of AUC of ROC of PI is 1-1 [1]. It is extremely unlikely that the observed sample parameter will depict the population parameter with such outstanding confidence.

This comment refers to the article available online at https://doi. org/10.1007/s00540-019-02652-2.

Priyam Saikia

saikia.priyam80@gmail.com

1 Department of Anaesthesiology and Critical Care, Gauhati Medical College and Hospital, Guwahati, Assam 781032, India
4. Sensitivity and specificity values observed in a sample are estimates for the corresponding population parameters. Thus, the authors are expected to report their CI.

\section{Compliance with ethical standards}

Conflict of interest None to declare.

\section{References}

1. Mowafy SMS, Abd Ellatif SE. Transcranial Doppler role in prediction of post-dural puncture headache in parturients undergoing elective cesarean section: prospective observational study. J Anesth. 2019;33:426-34.

2. Hajian-Tilaki K. Sample size estimation in diagnostic test studies of biomedical informatics. J Biomed Inform. 2014;48:193-204.

3. Serra-Serra V, Kyle PM, Chandran R, Redman CW. Maternal middle cerebral artery velocimetry in normal pregnancy and postpartum. BJOG. 1997;104:904-9.

Publisher's Note Springer Nature remains neutral with regard to jurisdictional claims in published maps and institutional affiliations. 